
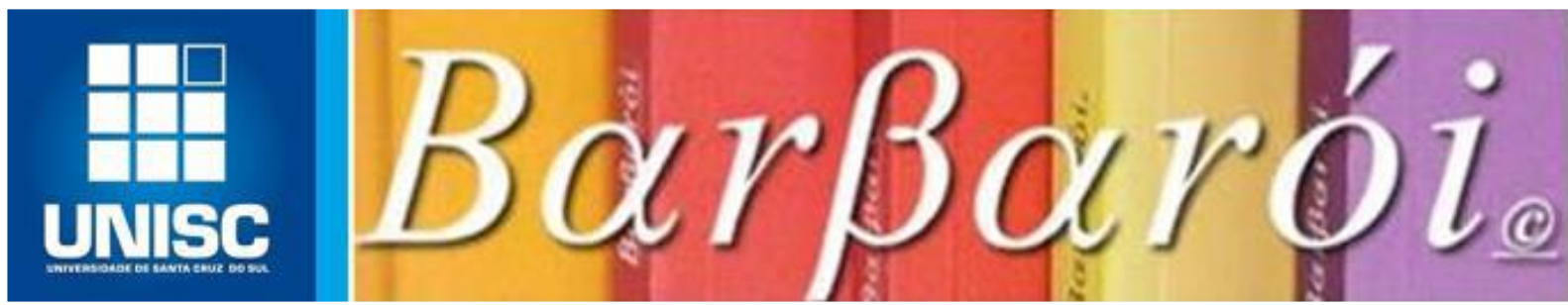

\title{
CONJUGANDO MÉTODOS CLÍNICO E EMPÍRICO NO ESTUDO DE CASO DE INTERRUPÇÃO PRECOCE EM PSICOTERAPIA PSICANALÍTICA Compreendendo a interrupção precoce em psicoterapia psicanalítica
}

DOI: http://dx.doi.org/10.17058/barbaroi.v0i0.9392

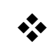 \\ Pricilla Braga Laskoski \\ Universidade Federal do Rio Grande do Sul-UFRGS - Brasil \\ Lívia Fração Sanchez \\ Universidade do Vale do Rio dos Sinos - UNISINOS - Brasil \\ Clarice Kern Ruaro \\ Instituto de Ensino e Pesquisa em Psicoterapia-IEPP-Brasil
}

Rafael Stella Wellausen

Hospital de Clínicas de Porto Alegre-HCPA-Brasil

Silvia Pereira da Cruz Benetti

Universidade do Vale do Rio dos Sinos - UNISINOS - Brasil

Fernanda Barcellos Serralta

Universidade do Vale do Rio dos Sinos - UNISINOS - Brasil

\section{Resumo}

O objetivo deste estudo é conjugar métodos clínico e empírico de avaliação do processo psicoterapêutico para a compreensão dos fatores associados à interrupção precoce em um caso de psicoterapia psicanalítica. Os participantes foram uma díade terapêutica. A paciente é jovem adulta, atendida em uma clínica-escola, por um período de três meses. A psicoterapeuta registrou sua impressão das sessões e sua compreensão dinâmica do caso. O método de estudo do caso foi análise sistemática por meio do Psychotherapy Process $Q$-Set (PQS) e do ShedlerWesten Assessment Procedure (SWAP). As sessões foram gravadas em vídeo e posteriormente codificadas com os instrumentos por avaliadores externos treinados. As características do processo indicam foco nos relacionamentos amorosos e nas situações de vida atual; a paciente trazia material significativo; tendia a concordar com as intervenções da psicoterapeuta e demonstrava se sentir entendida. A psicoterapeuta, por sua vez, mostrou empatia e adotou atitude de apoio, realizando intervenções exploratórias. A sessão 6 constituiu um momento de mudança na direção do processo. Os resultados fornecem elementos para a compreensão dos fatores relacionados com a interrupção, tais como o 
incremento da resistência e ambivalência por parte da paciente não devidamente abordados pela terapeuta.

Palavras-chave: abandono; psicoterapia psicanalítica; interrupção precoce; pesquisa em psicoterapia; processo terapêutico; pesquisa de processo.

\section{Introdução}

O processo de uma psicoterapia psicanalítica idealmente apresenta três fases distintas: inicial, intermediária e final. A etapa inicial tem como característica principal o estabelecimento de uma aliança terapêutica que sustente o trabalho com os conflitos que irá se desenvolver nas fases subsequentes. A fase intermediária é a mais longa, quando são explorados, examinados e resolvidos os sintomas e dificuldades emocionais do paciente. A etapa final, por sua vez, compreende o trabalho de elaboração de ansiedades decorrentes da separação paciente-terapeuta e ocorre após a avaliação de ambos acerca dos ganhos reais e mudanças obtidas em diversas áreas da vida do paciente no decorrer do processo (LUZ, 2015). Sabe-se, entretanto, que esse processo ideal nem sempre se completa e que frequentemente pacientes decidem sozinhos encerrar seus tratamentos de diversas formas e por diferentes razões (JUNG; SERRALTA; NUNES; EIZIRIK, 2013).

Considerando a existência de uma variada terminologia para designar a interrupção de uma psicoterapia antes que o trabalho terapêutico atinja os objetivos delimitados por paciente e psicoterapeuta - abandono, descontinuação prematura, término prematuro, término unilateral, entre outras - adotamos a expressão interrupção precoce para designar a interrupção unilateral do processo terapêutico por parte do paciente nas sessões iniciais de uma psicoterapia supostamente de longo prazo. A interrupção precoce em psicoterapia ocorre em um a cada cinco casos (SWIFT; GREENBERG, 2012), sendo as taxas maiores nos primeiros dois meses de tratamento (MARTINO; MENCHETTI; POZZI; BERARDI, 2012). Nas quatro primeiras sessões, o risco de o paciente abandonar o tratamento é muito elevado, e após a décima sessão esse risco cai drasticamente (URTIAGAet al., 1997). Gastaud e Nunes (2009), ao revisarem vários estudos sobre o tema, apresentam uma taxa de abandono em psicoterapia que varia entre 25 e $60 \%$ dos casos.

A psicoterapia psicanalítica de longo prazo tem como essência explorar os aspectos do self que não são totalmente conscientes, especialmente aqueles que são manifestados e potencialmente influenciados pela relação terapêutica (SHEDLER, 2010). Conforme Eizirik, Libermann e Costa (2008), a relação terapêutica é o veículo por meio do qual se processam os tratamentos psicoterápicos. O resultado de cada psicoterapia acaba por ser a soma das características individuais do paciente e do psicoterapeuta, das reedições de vivências Barbarói, Santa Cruz do Sul, n. 56, p.<208-222>,jan./jun. 2020 
passadas que eles aportam à situação atual e, por fim, da interação de todos esses elementos com a situação presente. Desta forma, tanto aspectos do paciente quanto do terapeuta bem como da relação estabelecida pela dupla são fatores que podem predispor o tratamento a uma interrupção precoce.

Altos índices de abandono em psicoterapia psicanalítica têm sido relacionados a fatores como o grau de motivação, expectativas de resultados, resistência, capacidade de insight e aliança terapêutica (HAUCK et al., 2007;MARTINO et al., 2012;MEYER et al., 2002).Um estudo qualitativo com um grupo de pacientes que abandonaram precocemente seus tratamentos de abordagem psicanalítica identificou diversos aspectos relacionados à interrupção, tais como: iniciar o tratamento por indicação de terceiros, apresentar maior resistência, expectativas de mais apoio, menor transferência positiva, mais queixas depressivas e experiências negativas com tratamentos anteriores. Na entrevista póstratamento, os participantes revelaram mais resistência durante o processo de psicoterapia (JUNG; SERRALTA; NUNES; EIZIRIK, 2014).

Considerando que a interrupção precoce consiste em um fenômeno complexo e multifatorial, acredita-se que uma abordagem ampla ao examinar o processo terapêutico possa contribuir de forma mais consistente para a sua compreensão. Nesse sentido, o presente estudo tem por objetivo conjugar o método clínico com métodos empíricos de avaliação do processo psicoterapêutico para ampliar a compreensão de um caso de interrupção precoce em psicoterapia psicanalítica. Por envolver a investigação sistemática da experiência humana em um contexto interpessoal (BUCCI, 2007), a pesquisa de processo em psicoterapia psicanalítica tem priorizado os estudos intensivos de caso. Estes possuem algumas semelhanças com os estudos de casos clínicos realizados por psicoterapeutas, mas apresentam maior rigor metodológico, aliando a subjetividade inerente à clínica com as exigências de objetividade, de validade e de replicabilidade das ciências empíricas (SERRALTA; NUNES; EIZIRIK, 2011). Nesse contexto, a combinação de avaliações qualitativas e quantitativas tem se mostrado bastante útil para a avaliação do processo psicoterapêutico, uma vez que permite contemplar, simultaneamente, a exploração das características idiossincráticas de cada caso e a magnitude da mudança (OGLES; LAMBERT; MASTERS, 1996).

\section{Método}

\section{Delineamento}

Estudo sistemático de caso único que consiste em um modelo de investigação que permite responder questões relevantes para a prática clínica por meio de evidências científicas Barbarói, Santa Cruz do Sul, n. 56, p.<208-222>,jan./jun. 2020 
(EDWARDS, 2007). Utiliza diferentes mecanismos na coleta dos dados para aprimorar o rigor metodológico, com procedimentos cuidadosos e sistemáticos, como a gravação em vídeo das sessões de psicoterapia para que se possa retornar aos dados sempre que desejável. Além disso, sugere-se que juízes independentes ao caso o analisem, através de diferentes instrumentos e por meio das gravações.

\section{Participantes}

Paciente. Lara (nome e algumas informações foram alterados para proteger a privacidade da paciente), 26 anos, ensino superior incompleto, casada e mãe de um menino pré-escolar, fruto de um relacionamento anterior. Buscou psicoterapia por desejar obter maior autoconfiança. Apresentava dificuldades conjugais, dentre elas o fato de o marido fazer uso abusivo de álcool, o que a incomodava bastante principalmente quando esse comportamento ocorria na presença do filho. Na primeira sessão, Lara afirmou que desejava se separar do marido, mas diz não ter coragem para isso. Acreditava que iria se sentir sozinha caso tomasse essa atitude, relatando muito medo dessa condição. Nunca passou longos períodos de tempo sem algum companheiro, iniciando novos relacionamentos imediatamente após o rompimento dos anteriores. Sobre a história pregressa, destaca-se que Lara não é filha biológica de seu pai (ela é fruto de um relacionamento anterior da mãe). Na infância, sentia que o pai adotivo era mais severo com ela do que com os outros filhos (estes biológicos). Tal configuração familiar acabou se repetindo na história recente da paciente, visto que seu atual marido também não é pai biológico de seu filho.

Terapeuta. A psicoterapeuta era mulher, psicóloga com pós-graduação em psicoterapia psicanalítica e 5 anos de experiência clínica.

Tratamento. A paciente foi atendida em psicoterapia psicanalítica com frequência semanal e duração de três meses, totalizando 14 sessões, das quais compareceu a 8 , sendo elas: $1^{\mathrm{a}}, 2^{\mathrm{a}}$, $3^{\mathrm{a}}, 5^{\mathrm{a}}, 6^{\mathrm{a}}, 7^{\mathrm{a}}, 8^{\mathrm{a}}$ e $14^{\mathrm{a}}$ sessão. Os atendimentos aconteceram no ambulatório de uma instituição de formação de psicoterapeutas psicanalíticos da cidade de Porto Alegre, RS.

\section{Instrumentos}

\section{Psychotherapy Process Q-Set (PQS)}

É um instrumento panteórico aplicável à hora terapêutica gravada em áudio e/ou vídeo (unidade de análise) cujo objetivo é realizar uma descrição detalhada, abrangente e quantificável dos elementos do processo terapêutico (JONES, 2000). O PQS é composto por 100 itens que incluem variáveis do paciente, do terapeuta e da interação entre ambos. O instrumento utiliza escala positiva de 9 pontos para classificar os itens em conformidade com Barbarói, Santa Cruz do Sul, n. 56, p.<208-222>,jan./jun. 2020 
a distribuição normal, sendo composto de uma planilha eletrônica acompanhada de um manual explicativo com descrições dos itens. Os juízes previamente treinados estudam o material da hora terapêutica e distribuem os itens em 9 categorias, variando num continuum que vai do menos característico (categoria 1) ao mais característico (categoria 9). A distribuição forçada é típica da metodologia Q e faz com que os juizes tenham que buscar o melhor arranjo para descrever os fenômenos, avaliando cada item em relação aos demais, o que requer tempo e reflexão. Com isso, evita-se efeitos de halo positivo e negativo (JONES, 2000; JONES; HALL; PARKE, 1991).

A versão original do PQS apresenta boa fidedignidade interavaliadores, validade de constructo e validade discriminante e ausência de estrutura fatorial (JONES; CUMMING; HOROWITZ, 1988). A versão em Português do Brasil (SERRALTA; NUNES; EIZIRIK, 2007), apresenta equivalência semântica com o instrumento original em inglês. Os coeficientes de fidedignidade entre avaliadores previamente treinados são comparáveis com os obtidos com o instrumento original.

Shedler-Westen Assessment Procedure - SWAP-200

É um instrumento desenvolvido para avaliar patologias de personalidade. É composto de 200 sentenças que expressam aspectos comportamentais, cognitivos, afetivos, relacionais, entre outros, dos pacientes com transtorno de personalidade (TP) (SHEDLER; WESTEN, 1998). Para cada uma das sentenças, o paciente recebe uma classificação T (de 0 a 7 ) que vai desde itens não-descritivos a respeito do paciente (0) até os altamente descritivos (7). Os pacientes que alcançarem escores $\mathrm{T}$ superiores a 60 alcançam determinado protótipo e recebem um diagnóstico de Transtorno de Personalidade (TP). O SWAP-200 possui 12 perfis hipotéticos, prototípicos para cada um dos TP, obtidos através de análises fatoriais Q, que irão configurar as Síndromes clínicas prototípicas do SWAP-200. Ao preencher critérios para qualquer um destes diagnósticos, é possível se obter um dos 10 diagnósticos de TP do DSM5. A estrutura fatorial do SWAP-200 abrange ainda 12 traços (fatores) que indicam áreas importantes do funcionamento, tais como: índice de saúde psicológica, psicopatia, hostilidade, narcisismo, desregulação emocional, disforia, orientação esquizóide, obsessividade, transtorno do pensamento, conflitos edípicos, dissociação e conflito sexual.

\section{Procedimentos}

Este estudo é derivado de um estudo mais amplo sobre processo terapêutico em psicoterapia psicanalítica. O protocolo do estudo foi aprovado pelo Comitê de Ética da 
universidade de origem do mesmo. Os participantes assinaram o Termo de Consentimento Livre e Esclarecido autorizando os procedimentos da pesquisa.

A coleta de dados (gravação em vídeo das sessões) ocorreu em uma clínica de uma instituição de ensino na cidade de Porto Alegre, RS. As sessões foram posteriormente codificadas com o PQS por duplas de avaliadores externos independentes treinados neste procedimento. A concordância entre avaliadores foi obtida através do coeficiente de correlação de Pearson, sendo adotado o ponto de corte de $r \leq 0,6$ para a inclusão de um terceiro avaliador. Para as análises subsequentes foi utilizada a média das avaliações dos dois avaliadores concordantes.

Para a descrição geral do processo, foi feito um ordenamento, a partir da média, dos itens mais e menos característicos. A avaliação da personalidade da paciente seguiu o procedimento padrão da SWAP-200. A avaliação clínica, realizada pela psicoterapeuta do caso, foi utilizada como forma complementar para a compreensão dos resultados.

\section{Resultados e Discussão}

Avaliação Clínica

A avaliação inicial realizada pela psicoterapeuta sugere que Lara demonstrou já nas primeiras sessões uma angústia de separação (indiscriminação/simbiose), de modo a não conseguir lidar com ausências e separações. Este foi um dos temas considerados pela terapeuta como importante de ser trabalhado ao longo da psicoterapia. Contudo, devido à interrupção precoce do tratamento pela paciente, isso não foi possível.

Além dos traços de dependência, Lara apresentava traços obsessivos. Estes eram demonstrados através do controle e da alta exigência da paciente consigo mesma e com os outros. Os traços obsessivos da paciente faziam com que ela tivesse um gasto energético alto e assim sentia-se cansada e sem poder finalizar suas tarefas. Um exemplo disso é a faculdade da paciente, que em um determinado momento teve que ser interrompida e, após alguns anos, retomada. A ambivalência sempre permeou a vida de Lara e foi em virtude de uma importante crise conjugal com risco iminente de separação que Lara buscou psicoterapia. Embora a relação conjugal apresentasse importantes componentes abusivos, a real possibilidade de uma separação mobilizava intenso temor de desorganização interna.

Como mecanismo de defesa, Lara utilizava em diversas situações a projeção colocando em seu marido grande parte de suas questões, assim como, deslocando chateações vividas em uma determinada situação para outra. Além disso, os usos da racionalização, 
negação e repressão eram evidentes como formas de evitar o contato com aspectos conflituosos de seu funcionamento psíquico.

Resultados da SWAP-200

A análise do caso pelo instrumento SWAP-200 gerou os seguintes gráficos (Figuras 1 e 2).

\section{Figura 1}

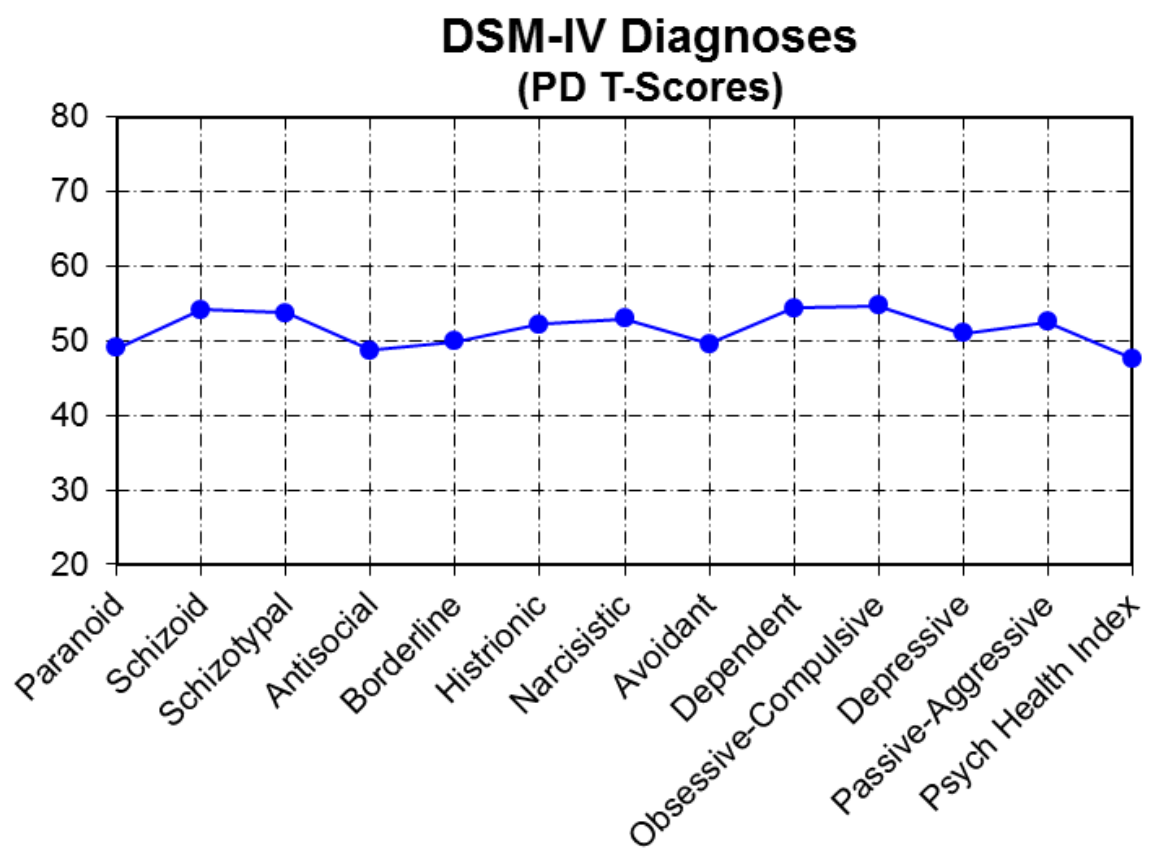

Figura 2

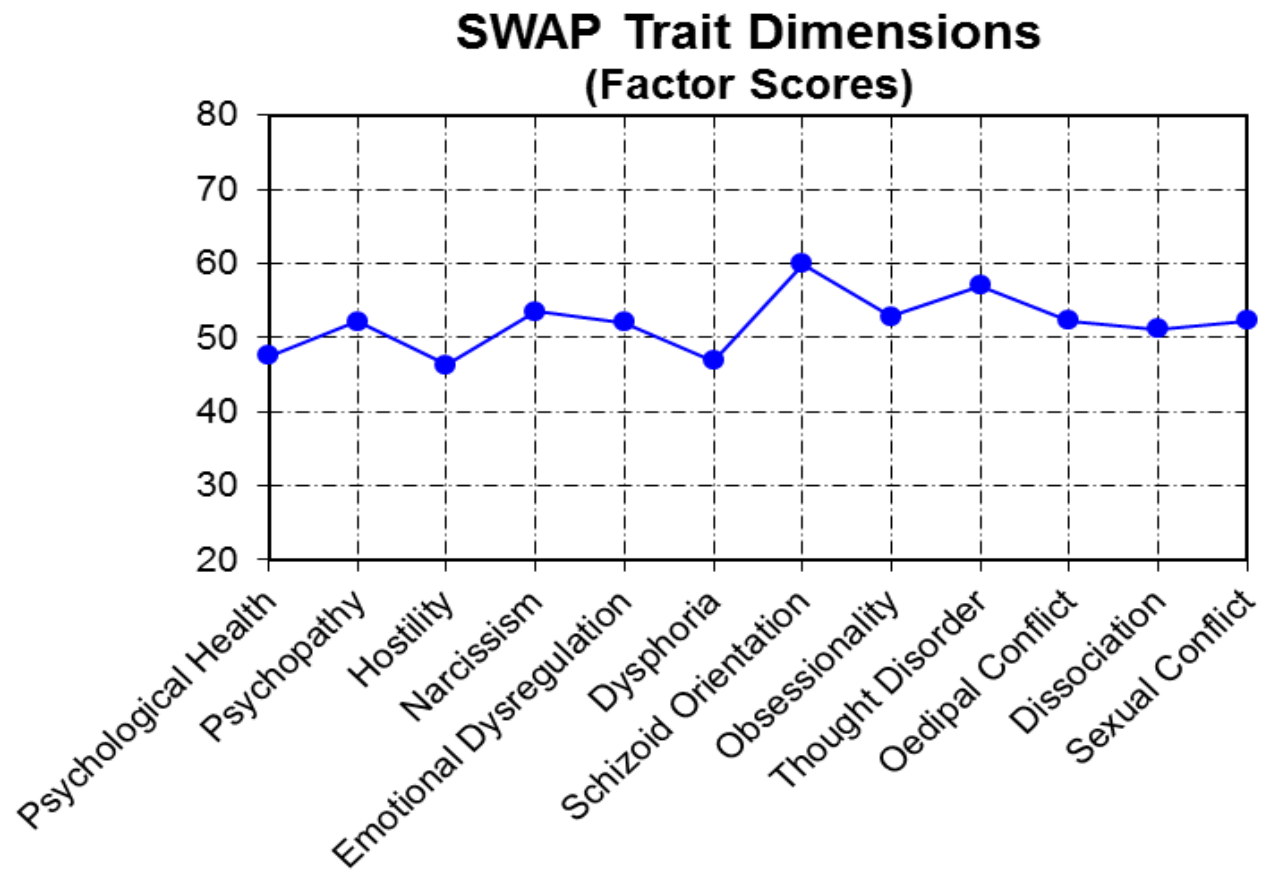

Barbarói, Santa Cruz do Sul, n. 56, p.<208-222>,jan./jun. 2020 
O primeiro gráfico (Figura 1) mede a correspondência entre o paciente e as descrições prototípicas da SWAP que representam os transtornos da personalidade de acordo com o DSM-5. Percebe-se que Lara não apresenta diagnóstico categorial de tais transtornos, pois nenhum dos escores atingiu o índice de 60 ou mais. No entanto, escores entre 55 e 59 garantem um diagnóstico de traços ou características do transtorno da personalidade correspondente. Assim, a partir do gráfico, nota-se que Lara apresenta traços dos transtornos da personalidade esquizóide, dependente e obsessivo-compulsiva.

O segundo gráfico (Figura 2), por sua vez, indica fatores da personalidade ou dimensões de traços derivados de análise fatorial do conjunto de itens da SWAP. Escores iguais ou superiores a 55 indicam fatores relevantes. Percebe-se que Lara apresenta tendências a orientação esquizoide bem como transtornos relacionados ao pensamento.

Os dados da SWAP-200 parecem corroborar o entendimento clínico da psicoterapeuta acerca do funcionamento psicodinâmico de Lara. A configuração dos traços de personalidade proposta pela SWAP-200 encontra respaldo na compreensão clínica e na história de vida da paciente. Lara teve que enfrentar importantes adversidades em seu desenvolvimento psicológico (abandono paterno, perdas etc.). É provável que, a fim de evitar sentimentos de insegurança, costume aderir rigidamente às rotinas tornando-se ansiosa quando elas são alteradas. Deste modo não surpreende que seja controladora e crítica em interações interpessoais, acreditando inclusive ser ou tornar-se perfeita em seu desempenho. $\mathrm{O}$ aspecto positivo é que tais características obsessivas podem fazer com que assuma de forma responsável e consciente seus compromissos e obrigações. Devido a sua rigidez de personalidade é comum que se sinta culpada ou que não se permita experimentar emoções prazerosas intensas (alegria, orgulho, excitação etc.) tendo ainda problemas com a expressão da raiva.

Em relação aos aspectos dependentes (ou infantis) de sua personalidade, fica evidente na avaliação que Lara parece temer ficar só, buscando a qualquer custo companhia, fantasiando encontrar um amor perfeito, ideal. Quando tem de enfrentar algumas situações estressantes é provável que exija a atenção de outra(s) pessoa(s) para regular o próprio afeto e se acalmar. Costuma sentir-se mal compreendida, maltratada e vitimizada. Nesses momentos, sente-se inadequada, inferior ou fracassada. A fim de evitar a solidão frequentemente se envolve em relacionamentos nos quais é emocional ou fisicamente abusada. Desse modo, é comum que seja sugestionável demonstrando dificuldades em tomar decisões e fazer escolhas. Por sentir-se frequentemente culpada, tem dificuldades de expressar ou reconhecer a própria raiva, porém quando o faz, é de modo indireto e passivo (esquecendo, cometendo enganos Barbarói, Santa Cruz do Sul, n. 56, p.<208-222>,jan./jun. 2020 
etc.). Fica claro que falta a Lara uma imagem estável dela mesma ou de quem gostaria de ser (seus valores objetivos e atitudes são determinados conforme a situação em que se encontra).

Por ter um funcionamento mais infantilizado e dependente, tem dificuldade em compreender o comportamento das outras pessoas, interpretando mal ou se atrapalhando com as ações alheias. Seu comportamento menos maduro faz com que tenha pouco ou nenhum interesse em ter relações sexuais com parceiros.

\section{Resultados do $P Q S$}

As características gerais do processo, obtidas através do PQS, indicam que a paciente é clara e organizada em suas verbalizações (PQS 54), traz material significativo às sessões (PQS 88) e de modo geral sente-se ajudada (PQS 95). A terapeuta é sensível aos sentimentos da paciente e empática ao material trazido por ela (PQS 6) e sua atitude é de aceitação nãocrítica (PQS18). As intervenções da terapeuta buscam facilitar as verbalizações da paciente (PQS 3) e solicitar mais informações a respeito de questões que possam não ter sido bem compreendidas (PQS 31). A paciente mostra-se ansiosa ou tensa (PQS 7) e fala sobre sentimentos de estar próxima ou precisando de alguém (PQS 33). Terapeuta e paciente exploram os temas dos relacionamentos amorosos (PQS 64) e interpessoais (PQS 63) da paciente, bem como a sua autoimagem (PQS 35) no contexto da sua vida atual (PQS 69).

Realizamos uma análise exploratória da variação dos itens do PQS obtidos ao longo do processo. Verificamos que na sessão 5 ocorreu uma mudança na direção do tratamento, indicada principalmente pelo aumento dos afetos negativos (PQS 26) e da resistência (PQS 58). Essa mudança está ilustrada nas Figuras 3 e 4, respectivamente.

\section{Figura 3}

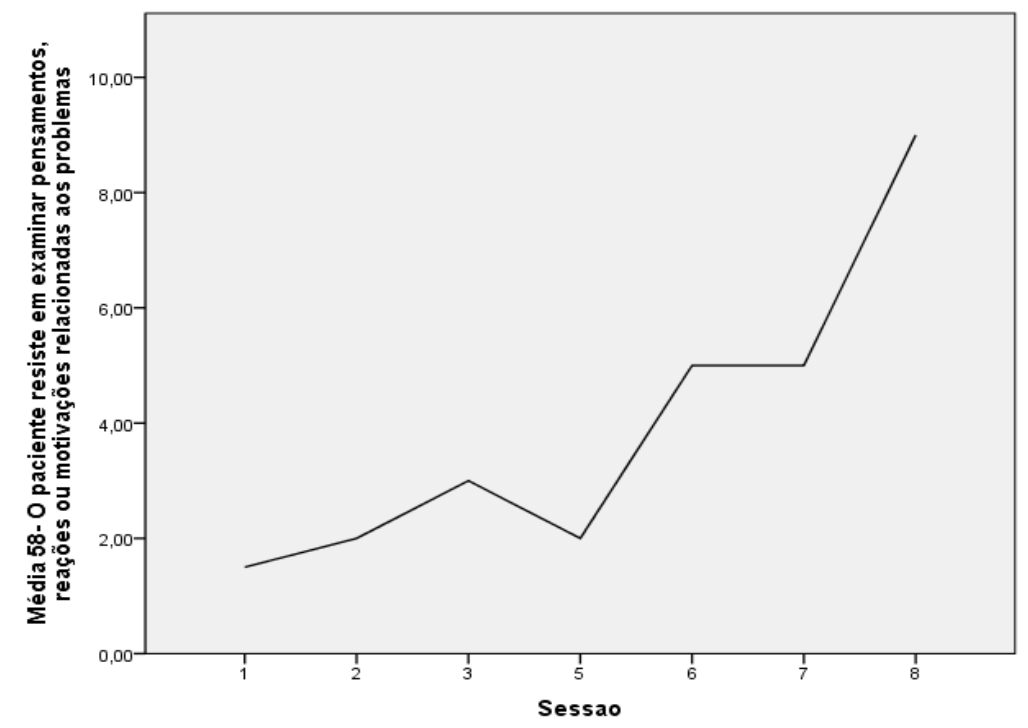




\section{Figura 4}

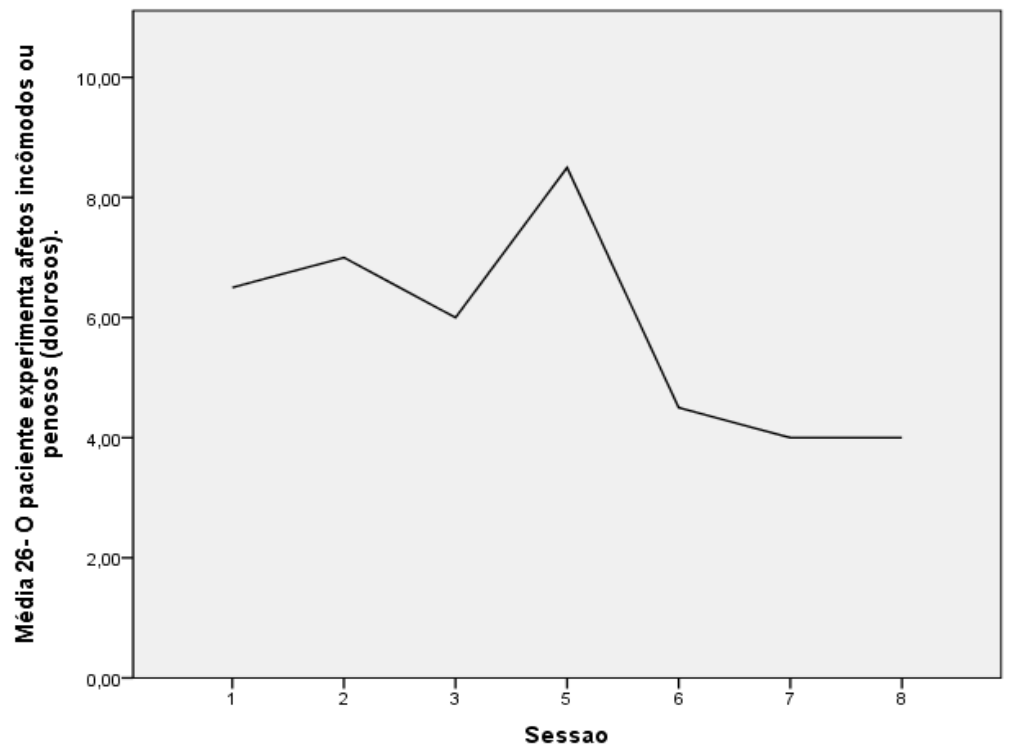

Procedemos a uma análise clínico-qualitativa das sessões 5 e 6 para compreender sua dinâmica e identificar os fenômenos que poderiam ter contribuído para o aumento da resistência e, consequentemente, para a interrupção precoce do tratamento. A paciente inicia a sessão 5 menos defensiva, mais deprimida e trazendo experiências conjugais dolorosas que impulsionavam-na a tomar uma posição mais ativa em relação ao seu casamento. $\mathrm{O}$ rompimento do laço conjugal parece iminente. Mostra-se mais próxima da terapeuta, demonstrando desejo de ser ajudada. Na sessão seguinte (sessão 6), está distanciada afetivamente das problemáticas trazidas ao tratamento e não parece contar com a terapeuta para a resolução destas.

Ao buscar compreensão do movimento da paciente e do tratamento ocorrido nessas duas sessões, foi possível perceber que, em ambas, a paciente mesmo se mostrando mais sintonizada com seus afetos, menos defensiva e mais deprimida (sessão 5), apresenta um padrão de oscilação afetiva. Reconhece suas dificuldades, mas, poucos instantes depois, se arma defensivamente por meio de racionalizações que busquem justificar sua permanência nas mesmas situações, retornando a uma posição passiva e vitimizada. Lara parece questionar suas próprias percepções como uma forma de não entrar em contato com a realidade de abusos que vive na atual relação conjugal. Tal movimento pode ser entendido como uma forte resistência da paciente em entrar em contato com seu mundo interno, seus conflitos e suas faltas afetivas. Compreender seu padrão relacional pode levar a um questionamento também acerca dos objetos com os quais a paciente se relacionou ao longo de sua vida - seus primeiros 
objetos de apego, o que é sentido por Lara como uma ameaça e consequentemente algo a ser evitado.

Quando há a presença de grande ambivalência nos momentos iniciais de um tratamento, e se esta não é efetivamente sinalizada e abordada pela terapeuta, o que pode ocorrer é um processo defensivo de dissociação por parte da paciente: o reconhecimento da angústia, da dor, bem como o desejo de mudança podem ser projetados no terapeuta, ou na terapia em si. Uma vez projetado em outro objeto, o paciente fica "livre" de entrar em contato com tais aspectos, e o terapeuta passa a ser um objeto persecutório, do qual o paciente precisa se desvencilhar. Esta configuração defensiva parece ter sido utilizada por Lara no tratamento, no momento em que passou a faltar a várias sessões (seis no total) até interrompê-lo definitivamente. A análise clínica das sessões 5 e 6 sugere que a ambivalência da paciente não foi um aspecto abordado pela terapeuta, podendo ser um fator contribuinte para seu término precoce.

\section{Considerações finais}

Este estudo conjugou métodos clínico e empírico com vistas a construir uma compreensão mais ampla do abandono precoce em psicoterapia psicanalítica. Nesse sentido, percebe-se que a conjugação dos instrumentos PQS e SWAP 200 com o método clínico podem ser complementares. Os instrumentos permitem uma verificação mais clara e objetiva dos fenômenos que acontecem dentro das sessões de psicoterapia. O PQS auxiliou a indicar um momento chave do tratamento (sessão 5) que pode ser melhor compreendido e analisado através do olhar clínico. Essa indicação de uma mudança (ilustrada nos gráficos 1 e 2) possibilitou o mapeamento do processo até então, evidenciando dessa forma pontos de alerta cruciais para a continuidade do tratamento ou a sua interrupção. Com base em tais pontos (a saber, os focos de resistência e ambivalência observados nas sessões 5 e 6), pode-se compreender clinicamente o fenômeno que escapa de uma dimensão mais objetiva fornecida pelos instrumentos.

O conjunto da análise realizada permite inferir que Lara deseja e teme aproximar-se de seu mundo interno e, por conseguinte, da terapeuta. Na sessão 5 há um ápice nesta aproximação e a aliança terapêutica está mais fortalecida. A paciente se permite experimentar afetos dolorosos até então negados, relacionados com a sua situação de vida atual. A paciente toma consciência da necessidade de efetuar mudanças reais internas e externas. A partir deste ponto, na sessão 6 e nas seguintes, há indícios de que o temor das mudanças parece 
preponderar sobre o mal-estar subjetivo e o desejo de se tratar culminando assim no incremento da resistência até a interrupção do tratamento.

Haja vista a ocorrência de altas taxas de abandono em psicoterapia e a frustração inerente a esse fenômeno tanto no que diz respeito ao paciente, que se sente desesperançoso com a possibilidade de vir a ser ajudado novamente, assim como, com os terapeutas que se sentem fracassados em seu trabalho, aponta-se a relevância e a necessidade de novos estudos sobre essa temática. Embora os resultados deste estudo correspondam apenas ao caso apresentado e generalizações não são possíveis a partir dele, espera-se contribuir para uma maior compreensão do fenômeno em questão e, consequentemente, para a realização de tratamentos mais efetivos e com menores taxas de interrupção precoce.

\title{
COMBINING CLINICAL AND EMPIRICAL METHODS FOR UNDERSTANDING A CASE OF PREMATURE INTERRUPTION IN PSYCHOANALYTIC PSYCHOTHERAPY
}

\author{
Understanding premature interruption in psychoanalytic psychotherapy
}

\begin{abstract}
This study aims to combine clinical and empirical methods of therapeutic process evaluation for understanding factors associated with premature interruption in psychoanalytic psychotherapy. The participants were a therapeutic dyad. The patient is a female young adult treated in an outpatient clinic of a psychoanalytic psychotherapy training institution. The treatment lasted three months, and the patient attended 8 sessions. The psychotherapist made records about her impressions of the sessions and about her understanding of the case dynamics. The case was analyzed systematically through the Psychotherapy Process Q-Set (PQS) and the Shedler-Westen Assessment Procedure (SWAP). Therapy sessions were videotaped and later coded by external trained raters. The general features of this process indicated that psychotherapy focused on romantic relationships and actual life situations; patient usually had brought significant materials; patient tended to agree with therapist's interventions, and appear to feel understood by the therapist. The therapist showed empathy and adopted a supportive attitude while conducting exploratory interventions. Session 6 was a turning point in the process direction. Results provide some elements for understanding process factors related to treatment interruption such as the increase of resistance and ambivalence on the part of the patient, which were not adequately addressed by the therapist.
\end{abstract}

Keywords: dropout; psychoanalytic psychotherapy; psychodynamic psychotherapy; psychotherapy research; therapeutic process; process research.

\section{REFERÊNCIAS}

BUCCI, W. Pesquisa sobre processo. In PEARSON, E.;COOPER, A. M.;GABBARD, G. O. (Orgs.). Compêndio de Psicanálise. Porto Alegre: Artmed, 2007. p. 305-319.

EDWARDS, D. J. A. Collaborative versus adverial stances in scientific discourse: Inplications for the role of sistematic case studies in the development of evidence-based practice in psychotherapy. |versão eletrônica|. Pragmatic Case studies in Psychotherapy, 3 (1), 
6-34, 2007. Obtido em 9 de outubro de 2011 do World Wide Web:

http://pcsp.libraries.rutgers.edu./index.php/pcsp/article/view/892/2260

EIZIRIK, C. L., LIBERMANN, Z. \& COSTA, F. A relação terapêutica: transferência, contratransferência e aliança terapêutica. In: Cordioli, A. C. (Org.) Psicoterapias: abordagens atuais. Porto Alegre: Artmed, 2008.

GAUSTAUD, M. B.; NUNES, M. L. T. Preditores de Abandono de Tratamento na Psicoterapia Psicanalítica de Crianças. Revista de Psiquiatria do Rio Grande do Sul (Impresso), n. 31, p. 13-23, 2009.

HAUCK, S.; KRUEL, L.; SORDI, A.; SBARDELLOTTO, G.; CERVIERI, A.; MOSCHETTI, L.; SCHESTATSKY, S.; CEITLIN, L. H. Fatores associados a abandono precoce do tratamento em psicoterapia de orientação analítica. Revista de Psiquiatria do Rio Grande do Sul, n. 29(3), p. 265-273, 2007.

JONES, E. E. Therapeutic Action: A guide to psychoanalytic therapy. New Jersey: Aronson, 2000 .

JONES, E. E.; CUMMING, J. D.; HOROWITZ, M. J. Another look at the nonspecific hypothesis of therapeutic effectiveness. Journal of Consulting and Clinical Psychology, $\mathrm{n}$. 56(1), p. 48-55, 1988.

JONES, E. E.; HALL, S.; PARKE, L. A. The process of change: The Berkeley Psychotherapy Research Group. In: Beutler, L.; Crago, M. (Eds.). Psychotherapy research: An international review of programmatic studies. Washington, DC: American Psychological Association, p. 98-106, 1991.

JUNG, S. I.; SERRALTA, F. B.;NUNES, M. L T.; EIZIRIK, C. L. Beginning and end of treatment of patients who dropped out of psychoanalytic psychotherapy. Trends in Psychiatry and Psychotherapy, n. 35, p. 181-190, 2013.

JUNG, S. I.; SERRALTA, F. B.;NUNES, M. L T.; EIZIRIK, C. L. Momentos distintos no abandono da psicoterapia psicanalítica. Jornal Brasileiro de Psiquiatria (UFRJ. Impresso), n. 63, p. 133-141, 2014.

LAPLANCHE, J.; PONTALIS, J. B. Vocabulário da Psicanálise. São Paulo: Martins Fontes, 2004.

LUZ, A.B. Fases da psicoterapia. In: EIZIRIK, C. L.;AGUIAR, R. W.; SCHESTATSKY, S. S. (Orgs.). Psicoterapia de Orientação Analítica: fundamentos teóricos e clínicos. Porto Alegre: Artmed, 2015. p. 249-266.

MARTINO, F.; MENCHETTI, M.; POZZI, E.; BERARDI, D. Predictors of dropout among personality disorders in a specialist outpatients psychosocial treatment: a preliminary study. Psychiatry and Clinical Neurosciences, n. 66, p. 180-186, 2012.

MEYER, B.; PILKONIS, P.; KRUPNICK, J.; EGAN, M.; SIMMENS, S.; SOTSKY, S. Treatment expectancies, paciente alliance and outcome: Further analyses from the national 
institute of mental health treatment of depression collaborative research program. Journal of Consulting and Clinical Psychology, n. 70(4), p. 1051-1055, 2002.

OGLES, B. M.; LAMBERT, M. J.; MASTERS, K. S. Assessing outcome in clinical practice. Boston: Allyn and Bacon, 1996.

SERRALTA, F. B.; NUNES, M. L. T.; EIZIRIK, C. L. Elaboração da versão em português do Psychotherapy Process Q-Set. Revista de Psiquiatria do Rio Grande do Sul, n. 29(1), p. 44-55, 2007.

SERRALTA, F, B.; NUNES, M. L. T.; EIZIRIK, C. L. Considerações metodológicas sobre o estudo de caso na pesquisa em psicoterapia. Estudos de Psicologia (Campinas), n. 28(4), p. 501-510, 2011.

SHEDLER, J. The efficacy of pshycodynamic pshycoteraphy. American Psychologist, 65(2), 98-109, 2010.SHEDLER, J., \& WESTEN, D. Refining the measurement of axis II: a Q-sort procedure for assessing personality pathology. Assessment, n. 5(4), p. 333-353, 1998.

SWIFT, J. K.; GREENBERG, R. P. Premature discontinuation in adult psychotherapy: A meta-analysis. Journal of Consulting and Clinical Psychology, n. 80(4), p. 547-559, 2012.

URTIAGA, M. E.; ALMEIDA, G.; VIANNA, M. E. D.; SANTOS, M. V.; BOTELHO, S. Fatores preditivos de abandono em psicoterapias. Um estudo na Clínica Sérgio Abuchaim. Jornal Brasileiro de Psiquiatria, n. 6(5), p. 279-283, 1997.

Data de recebimento: $23 / 03 / 2017$

Data de aceite: 01/06/2020

\section{Sobre os autores:}

Pricilla Braga Laskoski é psicóloga, especialista em Psicoterapia Psicanalítica, mestre em Filosofia pela PUCRS, doutora e atualmente pós-doutoranda em Psiquiatria e Ciências do Comportamento pela UFRGS.

Livia Fração Sanchez é psicóloga, especialista em Psicoterapia de orientação Psicanalítica (IEPP), mestre em Psicologia Clínica (PUC-RS), doutora em Psicologia Clínica (Unisinos). Membro Aspirante da Sociedade Psicanalítica de Porto Alegre (SPPA).

Clarice Kern Ruaro é psicóloga, especialista em Psicologia Clínica pelo CFP e em Avaliação Psicológica pela UFRGS.

Rafael Stella Wellausen é psicólogo, mestre e doutor em Psicologia pela UFRGS. Psicólogo contratado do Hospital de Clínicas de Porto Alegre, RS.

Silvia Pereira da Cruz Benetti é psicóloga, especialista em Psicoterapia Psicanalítica, mestre em Psicologia da Educação pela UFRGS, doutora em Estudos da Criança e da Família pela Universidade Syracuse. 
Fernanda Barcellos Serralta é psicóloga, especialista em Psicoterapia Psicanalítica, mestre em Psicologia Clínica pela PUCRS, doutora em Ciências Médicas: Psiquiatria pela UFRGS, docente e pesquisadora no PPG Psicologia da UNISINOS. 